1993 IEEE INTERNATIONAL FREQUENCY CONTROL SYMPOSIUM

\title{
CROSS CORRELATION ANALYSIS IMPROVES TIME DOMAIN MEASUREMENTS
}

\author{
A. Lepek* and F. L. Walls \\ Time and Frequency Division \\ National Institute of Standards \& Technology \\ Boulder, CO 80303
}

\begin{abstract}
$\underline{\text { Abstract }}$
We introduce cross correlation-based variances $\left(\# \sigma^{2}\right)$ to estimate the Allan variance, the modified Allan variance, and the time variance in the presence of the measurement system noise. These variances substantially lower the short-term measurement noise floor; however, they require significantly more data. $\# \sigma^{2}$ is also used as a tool to analyze and improve the measurement system noise. $\# \sigma^{2}$ bears a precise relation to the 3 -cornered-hat $\sigma$. We have reduced the short-term noise floor for $\sigma_{\mathrm{y}}(1$ s) by a factor of 100 relative to our initial hardware and software configuration.
\end{abstract}

\section{$\underline{\text { Introduction }}$}

The resolution or noise floor in time-domain measurement systems is limited by added noise in the measurement equipment. In this paper we introduce several approaches to analyzing time-domain measurement data which use cross correlation to reveal data obscured by the measurement noise. Looking for estimation of the Allan variance in such an environment we use $\# \sigma_{\mathrm{y}}(\tau)$ (cross-sigma), $\# \bmod \sigma_{y}(\tau)$ (cross- mod sigma), and $\# \sigma_{x}(\tau)$ which have close correspondence to the traditional variances $\sigma_{y}(\tau), \bmod \sigma_{y}(\tau)$, and $\sigma_{x}(\tau)[1-3]$.

The principle is to measure the time differences between two clocks by means of two independent simultaneous measurements and then to cross-correlate and average them to reduce the contributions of the measurement noise. This approach yields substantial improvements in the short-term noise floor of the system. However, it requires significantly more data. In this paper we show how this analysis is applied to the NIST Extended Dual Mixer Time Difference Measurement

Contribution of the U.S. Government, not subject to copyright.

"Israel National Physical Laboratory, Hebrew University Campus, Jerusalem, Israel 91904
System $[4,5]$ (EDM system) resulting in a substantial improvement in the estimates of $\sigma_{y}(\tau), \bmod \sigma_{y}(\tau)$, and $\sigma_{x}(\tau)$. Following similar guidelines, other measurement configurations such as the common view GPS time transfer may benefit from this method as well.

We also address measures of correlation, extensions to 3-cornered-hat measurements, the use of multiple clocks, and estimation of uncertainties. By studying the nature of these parameters as a function of $\tau$ we were able to identify noise components that corresponded to physical processes within the measurement system. Once identified, we reduced their effect on the noise floor, resulting in a better measurement system.

\section{Definitions and Notations}

The Allan variance is defined as

$\sigma^{2}(\tau)=<\{\mathrm{X}(\mathrm{t})-2 \mathrm{X}(\mathrm{t}+\tau)+\mathrm{X}(\mathrm{t}+2 \tau)\}^{2}>/\left(2 \tau^{2}\right)=$ $\left\langle\delta^{2}\right\rangle=\langle\delta \cdot \delta>$

where $<>$ denotes ensemble average or sample average, $X(t)$ is a time series of time differences between two clocks spaces $\tau$ seconds apart, and $\delta$ is the normalized second difference. If $\tau$ is an integral number of $\tau_{0}$, that is, $\mathrm{n} \tau_{0}$ an overlapping average may be used by shifting the $\delta \cdot \delta$ terms by $\tau_{0}$.

If we have two series $a$ and $b$ of time differences measured at the same instants, we obtain two sigmas which are $\sigma^{2}(\mathrm{t})_{\mathrm{a}}=\left\langle\delta_{\mathrm{a}} \cdot \delta_{\mathrm{a}}\right\rangle$ and $\sigma_{\mathrm{y}}^{2}(\mathrm{t})_{\mathrm{b}}$ $=\left\langle\delta_{\mathrm{b}} \cdot \delta_{\mathrm{b}}\right\rangle$. We would like to think of these time series as originated from the same two clocks $i$ and $\mathrm{j}$ but from two measurement systems $\mathrm{A}$ and $\mathrm{B}$. For two such time series we define \# $\sigma_{y}(\tau)$ (read "crosssigma") as

$\# \sigma^{2}(\tau)_{\mathrm{ab}}=\left\langle\delta_{\mathrm{a}} \cdot \delta_{\mathrm{b}}\right\rangle$

where $a b$ indicates the origin of the input series $a$ and $\mathrm{b}$ of $\# \sigma_{\mathrm{y}}(\tau)$, and 
$\# \sigma_{\mathrm{y}}(\tau)_{\mathrm{ab}}=\operatorname{sign}\left(\left\langle\delta_{\mathrm{a}} \cdot \delta_{\mathrm{b}}\right\rangle\right)\left|\left\langle\delta_{\mathrm{a}} \cdot \delta_{\mathrm{b}}\right\rangle\right|^{1 / 2}$,

so the sign of $\left\langle\delta_{\mathrm{a}} \cdot \delta_{\mathrm{b}}\right\rangle$, which may be negative, is preserved. A change in the sign (as a function of $\tau$ ) in $\left\langle\delta_{\mathrm{a}} \cdot \delta_{\mathrm{b}}\right\rangle$ indicates a change in the causes for the correlation between the $\delta_{\mathrm{a}}$ and $\delta_{\mathrm{b}}$ time series.

$\# \sigma^{2}(\tau)$ bears a strong relation to the traditional covariance. A similar sigma was investigated by Groslambert et al. [6]. defined as

Similarly, the modified Allan variance is

$$
\bmod \sigma_{y}^{2}(\tau)=<\{\underline{X(t)-2 X(t+\tau)+X(t+2 \tau)}\}^{2}>/\left(2 \tau^{2}\right)=
$$$$
\langle\mu \cdot \mu>\text {, }
$$

where the underline denotes an average over the interval $r,<>$ denotes as average over the whole measurement time series, and $\mu$ is a normalized and averaged second difference. For the two time series, $\mathrm{a}$ and $\mathrm{b}$, we define $\#$ mod $\sigma_{\mathrm{y}}(\tau)$ (read cross-mod-sigma) by

$\# \bmod \sigma_{\mathrm{y}}^{2}(\tau)_{\mathrm{ab}}=\left\langle\mu_{\mathrm{a}} \cdot \mu_{\mathrm{b}}\right\rangle$, with

$\# \bmod \sigma_{\mathrm{y}}(\tau)_{\mathrm{ab}}=\operatorname{sign}\left(<\mu_{\mathrm{a}} \cdot \mu_{\mathrm{b}}>\right)\left|<\mu_{\mathrm{a}} \cdot \mu_{\mathrm{b}}>\right|^{1 / 2}$,

following same reasoning as for $\# \sigma$.

In the same fashion, we extend the definition of $\sigma_{x}(\tau)$ commonly called T-VAR or time variance to $\# \sigma_{x}(\tau)$, (read cross-T-VAR). That is,

$\# \sigma_{\mathrm{x}}^{2}(\tau)_{\mathrm{ab}}=\left\langle\epsilon_{\mathrm{a}} \cdot \epsilon_{\mathrm{b}}\right\rangle$, where [3]

$\epsilon=\tau / 3^{1 / 2}$.

To simplify notation and to cover all types of $\sigma$ and $\# \sigma$, we use $\mathrm{d}_{\mathrm{a}}$ for $\delta_{\mathrm{a}}, \mu_{\mathrm{a}}$ and $\epsilon_{\mathrm{a}}$. Then, for example, we have

$\# \sigma_{\mathrm{y}}^{2}(\tau)_{\mathrm{ab}}=\left\langle\mathrm{d}_{\mathrm{a}} \cdot \mathrm{d}_{\mathrm{b}}\right\rangle$

and

$\bmod \sigma_{\mathrm{y}}^{2}(\tau)_{\mathrm{a}}=\left\langle\mathrm{d}_{\mathrm{a}} \cdot \mathrm{d}_{\mathrm{a}}\right\rangle=\left\langle\mathrm{d}_{\mathrm{a}}^{2}\right\rangle$

So far, \# $\sigma$ was obtained by crossing $\mathrm{d}_{\mathrm{a}}$ and $d_{b}$, where each such second difference was obtained within one time series. It is possible to cross elements differently as will be clear from the following definition. We denote this type of $\# \sigma$ by $\#_{a} \sigma_{y}^{2}(\tau)$ (a denotes alternate for alternate crossing).

$$
\begin{aligned}
& \#_{a} \sigma_{y}^{2}(\tau)= \\
& <\left\{\mathrm{X}_{\mathrm{a}}(\mathrm{t}+2 \tau)-\mathrm{X}_{\mathrm{b}}(\mathrm{t}+\tau)-\mathrm{X}_{\mathrm{a}}(\mathrm{t}+\tau)+\mathrm{X}_{\mathrm{b}}(\mathrm{t})\right\}\left\{\mathrm{X}_{\mathrm{b}}(\mathrm{t}+2 \tau)-\right. \\
& \left.\mathrm{X}_{\mathrm{a}}(\mathrm{t}+\tau)-\mathrm{X}_{\mathrm{b}}(\mathrm{t}+\tau)+\mathrm{X}_{\mathrm{a}}(\mathrm{t})\right\}>/ 2 \tau \\
& =<\left\{\mathrm{X}_{\mathrm{a}}(\mathrm{t}+2 \tau)-\mathrm{X}_{\mathrm{a}}(\mathrm{t}+\tau)-\mathrm{X}_{\mathrm{b}}(\mathrm{t}+\tau)+\mathrm{X}_{\mathrm{b}}(\mathrm{t})\right\}\left\{\mathrm{X}_{\mathrm{b}}(\mathrm{t}+2 \tau)-\right. \\
& \left.\mathrm{X}_{\mathrm{b}}(\mathrm{t}+\tau)-\mathrm{X}_{\mathrm{a}}(\mathrm{t}+\tau)+\mathrm{X}_{\mathrm{a}}(\mathrm{t})\right\}>/ 2 \tau^{2} .
\end{aligned}
$$

Here we crossed $\mathrm{X}$ between the two series in the first line and $\mathrm{y}$ (the fractional frequency $[\mathrm{X}(\mathrm{t}+\tau)-\mathrm{X}(\mathrm{t})] / \tau)$ in the second line. The equality shows that it does not matter whether we cross $\mathrm{X}$ or $\mathrm{y}$; both methods result in the same $\#_{a} \sigma$. Experimentally we find that in most cases $\# \sigma$ is lower than $\#_{a} \sigma$ and the differences are small; therefore we will not discuss these alternatives any further.

\section{Measurement Setup}

The measurement system is the Extended Dual Mixer Time Difference Measuring System [4,5] used by NIST and some other laboratories for many years. This system is called the EDM system. Figure 1 is a block diagram of the system. Channel 1 is used as the system master. An offset frequency reference, generated from channel 1 , is mixed with all other input channels of same nominal frequency. (For these tests the input frequency was $5 \mathrm{MHz}$ and the beat frequency was $10 \mathrm{~Hz}$ ). The time differences between channel 1 and all other channels are obtained virtually simultaneously by means of time interval counters, one per channel. The counters are triggered by channel 1 , which provides the start pulse. The time differences between any two clocks (channels) are then computed from the comparisons with channel 1 .

To do the \# $\sigma$ analysis, we split each clock into two channels. This is done with a power splitter or a distribution amplifier. Figure 2 is a block diagram of the information flow in such a setup. The measurement blocks in this figure include both the hardware and the software functions. Thus for each two clocks we may obtain four time series. To be specific we choose two time series for a $\# \sigma$ analysis. We denote the channels due to clock $i$ as $c_{i a}$ and $c_{i b}$, and the channels due to clock $j$ as $c_{j a}$ and $c_{j b}$. Further, let us denote the time series (of second differences) $d_{a}$ as originating from comparing $c_{i a}$ with $\mathrm{c}_{\mathrm{ja}}$ and $\mathrm{d}_{\mathrm{b}}$ from comparing $\mathrm{c}_{\mathrm{ib}}$ and $\mathrm{c}_{\mathrm{jb}}$. We may calculate two additional time series $d_{c}$ and $d_{d}$ originating from the other two channel combinations. 


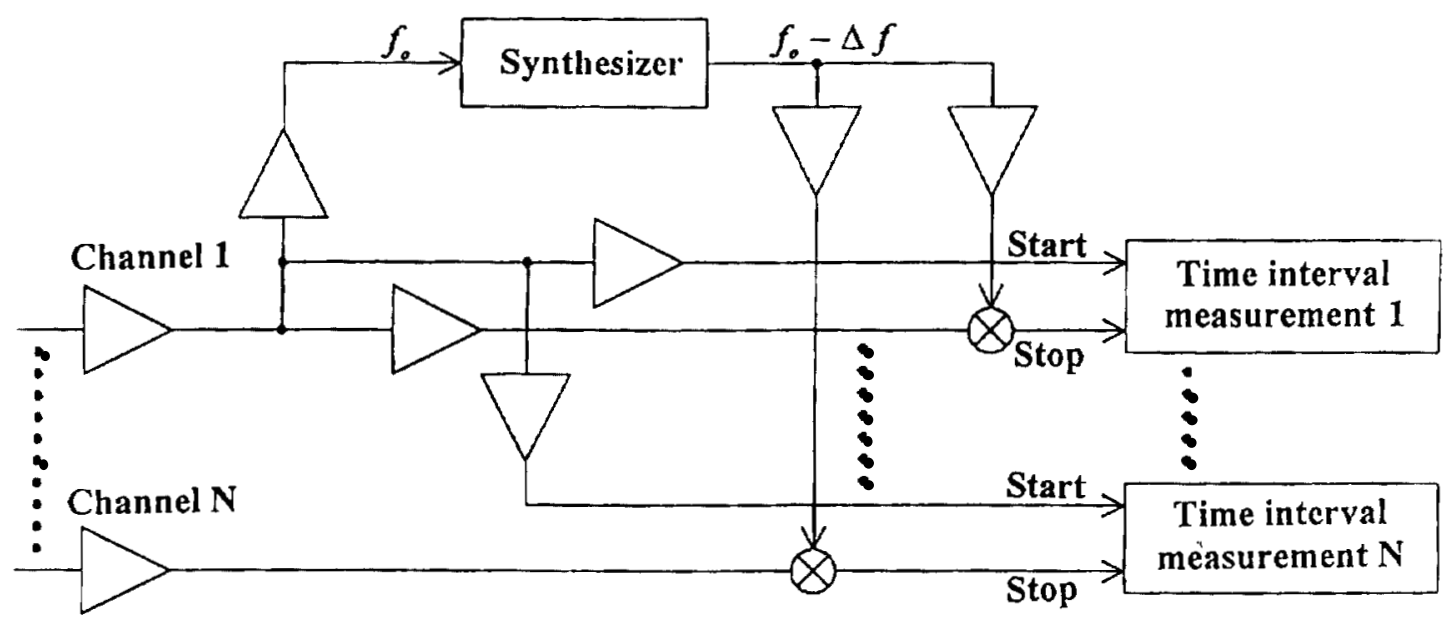

Figure 1. Block diagram of the extended dual mixer time difference measurement system.

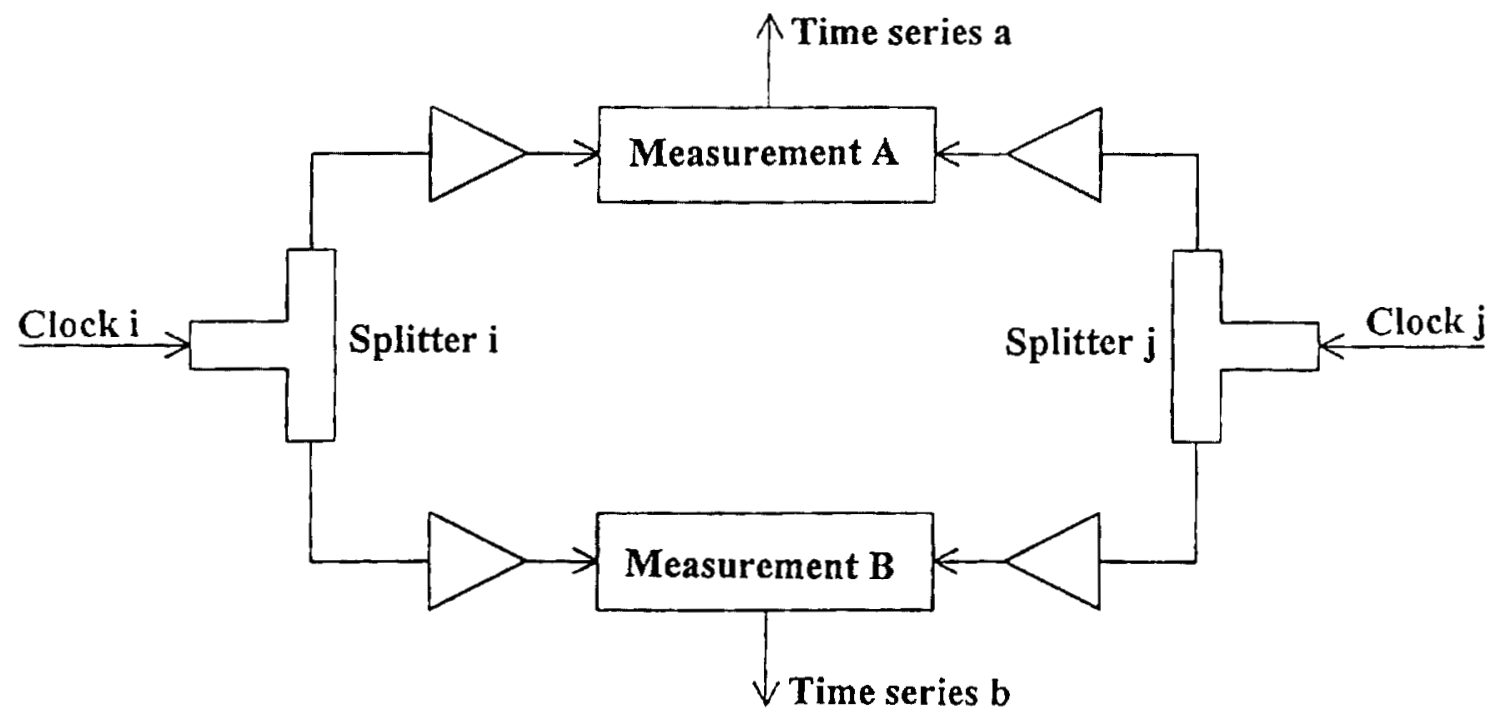

Figure 2. Information flow in the \# $\sigma$ setup. 
If we use all four channel combinations, we may use an extended $\# \sigma$ denoted as $\#_{4} \sigma$ defined as

$$
\#_{4} \sigma^{2}(\tau)=\left\langle\left(d_{a} \cdot d_{b} \cdot d_{c} \cdot d_{d}\right)^{1 / 2}\right\rangle .
$$

No change in the setup is required to use this equation since all four channels are already present once each clock is split. However, experimentally $\#_{4} \sigma$ has an advantage over $\# \sigma$ only in limited cases. The reason is that it is averaging the best and the worst channels, whereas when using $\# \sigma$ we can choose the best performing channels (having the least-correlated noise).

This setup may be extended to include more than two channels per clock. If the number $n$ of channels per clock is even, we may extend the definition of $\# \sigma$ to

$$
\#_{\mathrm{n}} \sigma_{\mathrm{y}}^{2}(\tau)=\left\langle\left(\mathrm{d}_{\mathrm{a}} \cdot \mathrm{d}_{\mathrm{b}} \cdot \mathrm{d}_{\mathrm{c}} \cdot \mathrm{d}_{\mathrm{d}} \ldots\right)^{2 / \mathrm{n}}\right\rangle
$$

A still different extension is to compare one clock to two different reference clocks. As will be shown, the $\# \sigma^{2}$ of this combination is exactly equivalent to the 3-cornered-hat Allan variance. Figure 3 shows a setup for 3-cornered-hat where clock $i$ is split into two channels, each of which is compared to two other clocks through two combinations.

\section{General Noise Relationships}

We may break down $d_{a}$ and $d_{b}$ so that $d_{a}=Q+A$ and $d_{b}=Q+B$, where $Q$ is the time series due to the clocks only (assuming ideal measurement) and $A$ or $B$ are due to the added noise in the measuring systems. The task is to estimate the Allan variance $\left\langle\mathrm{Q}^{2}\right\rangle$ in the presence of the added noise.

We note the following relations valid for any $\tau:$

$n_{\mathrm{a}}^{2}+\sigma_{\mathrm{b}}^{2}=\left\langle(\mathrm{Q}+\mathrm{A})^{2}+(\mathrm{Q}+\mathrm{B})^{2}\right\rangle=2\left\langle\mathrm{Q}^{2}\right\rangle+$

$2\langle\mathrm{Q}(\mathrm{A}+\mathrm{B})\rangle+\left\langle\mathrm{A}^{2}\right\rangle+\left\langle\mathrm{B}^{2}\right\rangle$,

$2 \# \sigma^{2}{ }_{\mathrm{ab}}=2\langle(\mathrm{Q}+\mathrm{A})(\mathrm{Q}+\mathrm{B})\rangle=2\left\langle\mathrm{Q}^{2}\right\rangle+$ $2\langle\mathrm{Q}(\mathrm{A}+\mathrm{B})\rangle+2<\mathrm{AB}\rangle$.

\section{Therefore:}

(1) If the measurement noise is low enough compared to the clocks that $\left.\left.\left.2<\mathrm{Q}^{2}\right\rangle \gg<\mathrm{A}^{2}\right\rangle+<\mathrm{B}^{2}\right\rangle$ (then $\left.2 \mathrm{Q}^{2} \gg 2<\mathrm{AB}\right\rangle$ ) and

$$
\left(\sigma_{\mathrm{a}}^{2}+\sigma_{\mathrm{b}}^{2}\right) / 2 \approx \# \sigma_{\mathrm{ab}}^{2},
$$

then $\# \sigma^{2}$ ab is a good estimate of the average Allan variances.

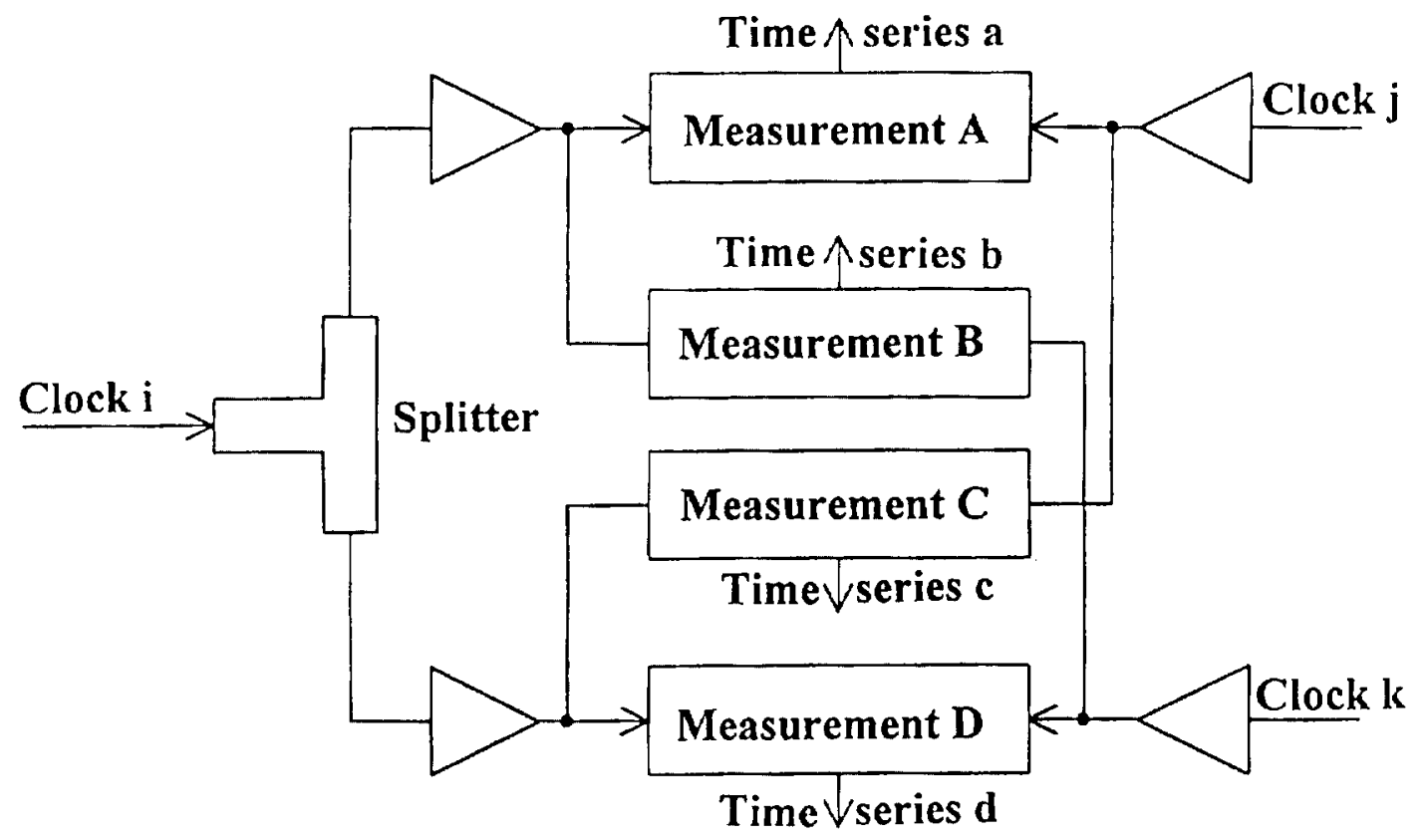

Fig. 3 Information flow in a $\# \sigma$ setup for 3-cornered-hat. 
(2) Since $\left.\left\langle\mathrm{A}^{2}\right\rangle+\left\langle\mathrm{B}^{2}\right\rangle \geq 2<\mathrm{AB}\right\rangle$ is always true, $\# \sigma^{2}$ ab is never higher than $\left(\sigma^{2}+\sigma_{b}^{2}\right) / 2, \# \sigma_{a b}^{2}$ is a better estimate of the Allan variance than the average $\left(\sigma_{\mathrm{a}}^{2}+\sigma_{\mathrm{b}}^{2}\right) / 2$ and practically it is better than the best of these two sigmas. Following the same reasoning, the difference

$$
\begin{aligned}
& \mathrm{D}^{2}(\tau)=\left(\sigma^{2}{ }_{\mathrm{a}}+\sigma_{\mathrm{b}}^{2}\right) / 2-\left|\# \sigma_{\mathrm{ab}}^{2}\right|=<\mathrm{A}^{2}+\mathrm{B}^{2}>/ 2- \\
& |<\mathrm{AB}>|
\end{aligned}
$$

is positive, is independent of $Q$, and characterizes the measurement system.

(3) To estimate the added noise by the system we connect the same clock to all inputs, then $\mathrm{Q}=0$. Therefore Eqs. (8) and (9) become

$\sigma_{\mathrm{a}}^{2}+\sigma_{\mathrm{b}}^{2}=\left\langle\mathrm{A}^{2}\right\rangle+\left\langle\mathrm{B}^{2}\right\rangle$,

$\left.2 \cdot \# \sigma^{2}{ }_{a b}=2<\mathrm{AB}\right\rangle$.

Therefore the lower is the correlation $\angle A B\rangle$ between $\mathrm{A}$ and $\mathrm{B}$, the higher is the ratio

$\left(\sigma^{2}{ }_{a}+\sigma_{b}^{2}\right) /\left|2 \cdot \# \sigma^{2}{ }_{a b}\right|=$

$\left.\left.<\mathrm{A}^{2}+\mathrm{B}^{2}\right\rangle|2|<\mathrm{AB}\right\rangle \mid$.

This ratio is a measure of the improvement in the noise floor due to the use of $\# \sigma$ instead of $\sigma$.

(4) We define the function $\mathrm{R}(\tau)$ as

$\mathbf{R}(\tau)=\# \sigma^{2}{ }_{a b} /\left(\sigma_{\mathrm{a}} \cdot \sigma_{\mathrm{b}}\right)$

When measuring the noise floor using the above method $Q=0$, and we obtain

$\mathrm{R}(\tau)=\langle\mathrm{A} \cdot \mathrm{B}\rangle /\left\{\left\langle\mathrm{A}^{2}\right\rangle \cdot\left\langle\mathrm{B}^{2}\right\rangle\right\}^{1 / 2}$.

Equation (20) shows that $R(\tau)$ is between 0 and 1 . The definition of $R(\tau)$ is similar to the definition of the Pearson correlation coefficient $r$ [7]. In statistics $\mathrm{r}^{2}$ is a measure of the correlated portion of $\sigma^{2}(\mathrm{x} 1)$ of the variable $x 1$ due to the linear regression of a second variable $\times 2$. Also, $\left(1-r^{2}\right)$ is a measure of the uncorrelated portion. Similarly we use $\mathrm{R}^{2}(\tau)$ as a measure of the correlation between different channels in the measurement system. We use $\mathbf{R}^{2}$ as a measure of the improvement due to the use of $\# \sigma^{2}$ and as a figure of merit reflecting the amount of correlated noise in the system.

(5) Since $\# \sigma^{2}{ }_{a b}=\left\langle\mathrm{d}_{\mathrm{a}} \cdot \mathrm{d}_{\mathrm{b}}\right\rangle, o^{2}{ }_{\mathrm{a}}=\left\langle\mathrm{d}_{\mathrm{a}} \cdot \mathrm{d}_{\mathrm{a}}\right\rangle$ and $\sigma_{b}^{2}=\left\langle\mathrm{d}_{\mathrm{b}} \cdot \mathrm{d}_{\mathrm{b}}\right\rangle$, the uncertainty in \# $\sigma$ (due to $\mathrm{i}$ : spread) is expected to be of the order of the uncertainties in $\sigma_{\mathrm{a}}$ and $\sigma_{\mathrm{b}}$. When using overlapping averages, a simple way to deal with uncertainties, especially when the noise is not white is to divide the time series interval (total number of points) into $\mathrm{N}$ equal intervals (we use four) and for each of them compute $\# \sigma_{\mathrm{ab}}, \sigma_{\alpha}$, and $\sigma_{\mathrm{b}}$. Then the standard deviations of $\# \sigma_{\mathrm{ab}}, \sigma_{\mathrm{a}}$ and $\sigma_{\mathrm{b}}$ are computed from these $\mathrm{n}$ values. They are then divided by $V_{\mathrm{n}}$ to obtain an estimate of the uncertainties $\# \mathrm{Er}_{\mathrm{ab}}, \mathrm{Er}_{\mathrm{a}}$ and $\mathrm{Er}_{\mathrm{b}}$. This method avoids any assumptions and the need to know the type of noise when estimating the uncertainty of $\sigma_{\mathrm{y}}$. The method used to compute the partial $\# \sigma_{\mathrm{ab}}, \sigma_{\mathrm{a}}$ and $\sigma_{\mathrm{b}}$ (overlapping intervals) is the same as for the complete series. An additional advantage of dividing

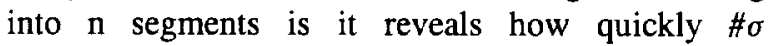
converges to a limit with the number of points. The uncertainty in $\# \sigma$ as a function of $\mathrm{n}$ can be used to detect this convergence.

(6) Application to 3-cornered-hat measurements. Comparing clock $\mathrm{i}$ to two other different clocks $j$ and $k$ results in two measured time series, $a=i j$ and $b=i k$. A third time series $c=j k$ may be computed from these two. We use here the ij notation to indicate the origin of the time series. As before,

$\sigma_{i j}^{2}=\left\langle\mathrm{d}_{\mathrm{ij}} \cdot \mathrm{d}_{\mathrm{ij}}\right\rangle$

the 3 corner hat $\sigma_{\mathrm{i}}$ is

$\sigma_{i}^{2}=<d_{i j} \cdot d_{i j}+d_{i k} \cdot d_{i k}-d_{j k} \cdot d_{j k}>/ 2$,

where $d_{j k}$ was calculated from the other series from

$d_{j k}=d_{i k}-d_{i j}$,

as is done in the EDM system. Substituting $d_{j k}$ yields

$$
\begin{aligned}
& \sigma_{i}^{2}=<d_{i j} \cdot d_{i j}+d_{i k} \cdot d_{i k}-\left(d_{i k}-d_{i j}\right) \cdot\left(d_{i k}-d_{i j}\right)>/ 2 \\
& =<d_{i j} \cdot d_{i k}+d_{i k} \cdot d_{i j}>/ 2=<d_{i j} \cdot d_{i k}>=\# \sigma_{i}^{2}(2 / R)
\end{aligned}
$$

where $\mathrm{ij} \_\mathrm{ik}$ indicate that the crossing is between $\mathrm{ij}$ and ik. This means that to get the 3-cornered-hatsigma we do not need to compute all three sigmas; we can use two time series and compute the cross-sigma.

Note that

$\sigma_{j}^{2}=\# \sigma^{2}{ }_{j i j k}=-\# \sigma^{2}{ }_{i j j} \mathbf{k}$, 
because

$\left\langle\mathrm{d}_{\mathrm{ji}} \cdot \mathrm{d}_{\mathrm{jk}}\right\rangle=-\left\langle\mathrm{d}_{\mathrm{ij}} \cdot \mathrm{d}_{\mathrm{jk}}\right\rangle$.

Usually, the 3-cornered-hat equation of $\sigma_{\mathrm{i}}$ is based on the assumption that the three sigmas are independent. This is not the case in the EDM system since the jk time series is calculated from the other two. This relationship occurs because all clock comparisons are derived by their comparison to clock 1 .

Going one step further, we can derive a 3cornered-hat for the $\# \sigma$. Let us assume that each one of the three clocks $i, j$, and $k$ is split into two channels. We can choose now two time series, for example $d_{i 1} j 1$ and $d_{i 2} j 2$, and compute \# $\sigma$ for them:

$\# \sigma^{2}{ }_{(i 1 j 1 \cdot i 2 j 2)}=\left\langle d_{i 1 j 1} \cdot d_{i 2 j 2}\right\rangle$.

Following Eq. (23) the 3-cornered-hat $\# \sigma^{2}$ for clock $\mathrm{i}$ is defined as

$\# \sigma^{2}{ }_{\mathrm{i}}=<\mathrm{d}_{\mathrm{i} 1 \mathrm{j} 1} \cdot \mathrm{d}_{\mathrm{i} 2 \mathrm{j} 2}+\mathrm{d}_{\mathrm{i} 1 \mathrm{k} 1} \cdot \mathrm{d}_{\mathrm{i} 2 \mathrm{k} 2}-$

$\mathrm{d}_{\mathrm{j} 1 \_\mathrm{k} 1} \cdot \mathrm{d}_{\mathrm{j} 2 \mathrm{k} 2}>/ 2$.

As before we obtain the $\mathrm{jk}$ series from the two other by

$\mathrm{d}_{\mathrm{j} 1 \mathrm{k} 1}=\mathrm{d}_{\mathrm{i} 1 \mathrm{k} 1}-\mathrm{d}_{\mathrm{i} 1 \mathrm{j} 2}$,

$\mathrm{d}_{\mathrm{j} 2 \mathrm{k} 2}=\mathrm{d}_{\mathrm{i} 2 \_\mathrm{k} 2}-\mathrm{d}_{\mathrm{i} 2 \mathrm{j} 2}$.

Substituting $d_{j 1 \_} k 1$ and $d_{j 2 \_} k$ yields

$\# \sigma^{2}$

$=<\mathrm{d}_{\mathrm{i} 1 \mathrm{j} 1} \cdot \mathrm{d}_{\mathrm{i} 2 \mathrm{j} 2}+\mathrm{d}_{\mathrm{i} 1 \mathrm{k} 1} \cdot \mathrm{d}_{\mathrm{i} 2 \mathrm{k} 2}-$

$\left(\mathrm{d}_{\mathrm{i} 1 \_\mathrm{k} 1}-\mathrm{d}_{\mathrm{i} 1} \mathrm{j} 2\right) \cdot\left(\mathrm{d}_{\mathrm{i} 2 \_\mathrm{k} 2}-\mathrm{d}_{\mathrm{i} 2} \mathrm{j} 2\right)>/ 2$,

$=<\mathrm{d}_{\mathrm{i} 1} \mathrm{j1} \cdot \mathrm{d}_{\mathrm{i} 2} \mathrm{k} 2 \mathrm{k}+\mathrm{d}_{\mathrm{i} 2} \mathrm{k} 1 \mathrm{~d}_{\mathrm{i} 2 \mathrm{j} 2}>/ 2$,

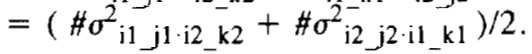

$\# \sigma^{2}$ is the average of the two $\# \sigma$ 's and is symmetrical in 1 and 2 .

Our previous discussions show that similar equations are obtained for $\# \bmod \sigma$ and $\# \sigma_{\mathrm{x}}$.

\section{$\underline{\text { Results }}$}

Analysis of the EDM system noise reveals several noise sources. These are counter quantization noise, instabilities in synthesizer mixer noise, digital level changes, distribution amplifiers, environmental effects (temperature and vibration), and spurious 60 $\mathrm{Hz}$ modulation. Some of these sources can be seen directly in the time series and some, such as digital levels, in statistical histograms of the time series. By analyzing $\# \sigma$, which is sensitive to correlated (or common) noise, we can trace the pollution of these types of noises in the system. It was impossible to use $\sigma$ as a guide because of it's much smaller sensitivity. Figure 4 shows an initial \# $\sigma$ plot of the system noise floor. The input frequency was $5 \mathrm{MHz}$ and the offset frequency is $10 \mathrm{~Hz}$ for Figures 4-8. In this figure we used the setup of Figure 2, where a and $b$ result from two chosen time series and all inputs originate from the same hydrogen maser. The relatively small differences between the $\# \sigma$ and $\sigma$ show that the correlated noise is high and probably accounts for most of the measurement noise. Using 10000 points for $\tau_{0}$, the uncertainties in this and the following graphs are at the level of few percent. An effort was made to cancel the correlated noise to as low a level as possible. This included changes in the offset synthesizer to lower the PLL time constants, replacement of noisy components and especially the mixers, isolation and filtering of the power supplies, and the use of power splitters instead of distribution amplifiers. We have found also that dc isolation of the incoming signals reduces measurement noise. Figure 5 shows the present state of the system noise floor obtained by injecting the same clock to all channels. Figure 6 shows the \#mod $\sigma$ analysis of the data of Figure 5. Note the sign change in $\# \sigma$ and \#mod $\sigma$ at about $8 \mathrm{~s}$ in Figures 5 and 6; this indicates a change in the causes of the correlations. $|\# \sigma|$ may be very low at the regions of sign change due to the balance of these causes, resulting in effective uncorrelated noise. This change is supported by Figure 7 where $\mathrm{R}^{2}(\tau)$ has two or three peaks in the correlated noise. The noise floor peaking around $\tau=30 \mathrm{~s}$ is probably due to the synthesizer's instability, as can be verified by changing components in the synthesizer. The improvement in noise floor is nearly a factor of 100 over the initial performance and seen in Figures 4-6.

Figure 8 shows the 3-cornered-hat \# $\sigma$ obtained for an active hydrogen maser splitting it (as clock $i$ in Figure 3) and crossing each of the two channels with two other hydrogen masers. We note the influence of the synthesizer's instability peaking around $\tau=30 \mathrm{~s}$. Comparing with Figure 4 shows that this measurement was impossible before the system improvements. 


\section{Initial Noise Floor for EDM}

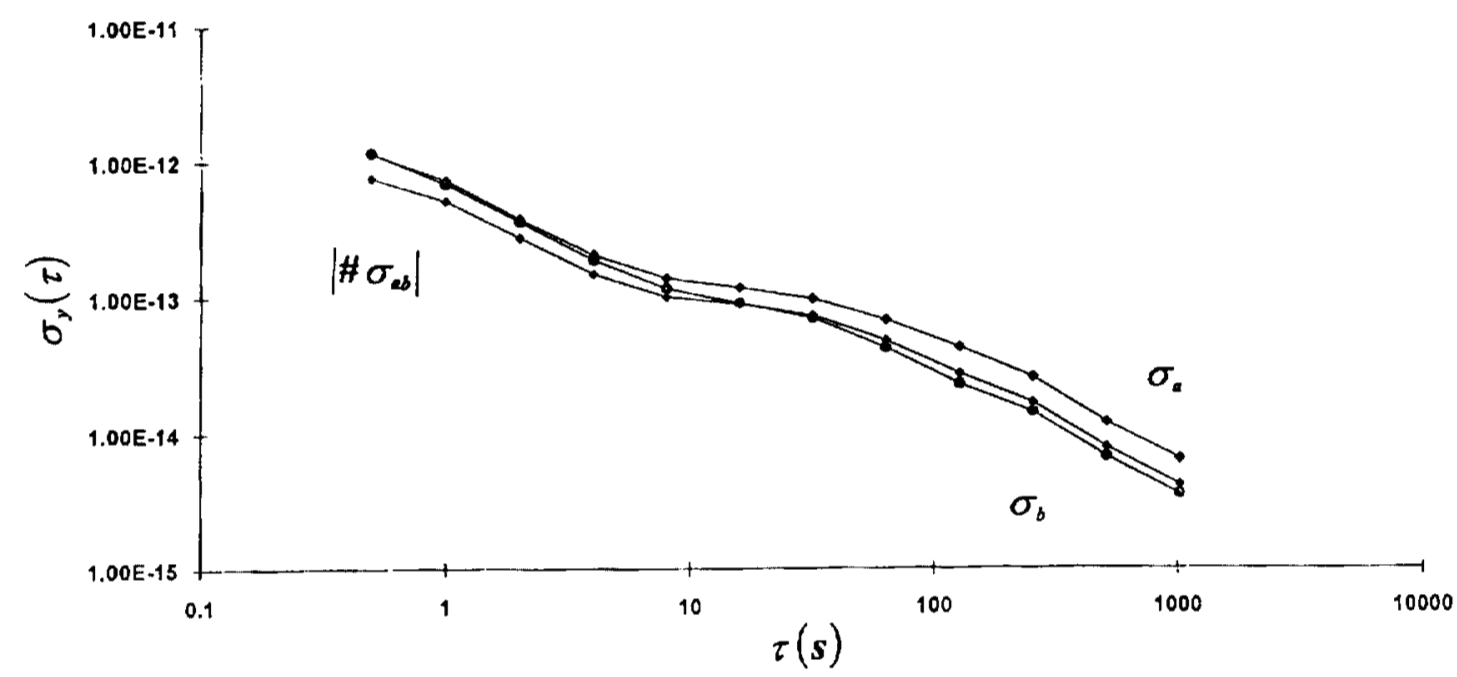

Figure 4. Initial noise floor for the NIST Extended Dual Mixer System (EDM) versus measurement time $\tau$.

Improved System Noise Floor for EDM

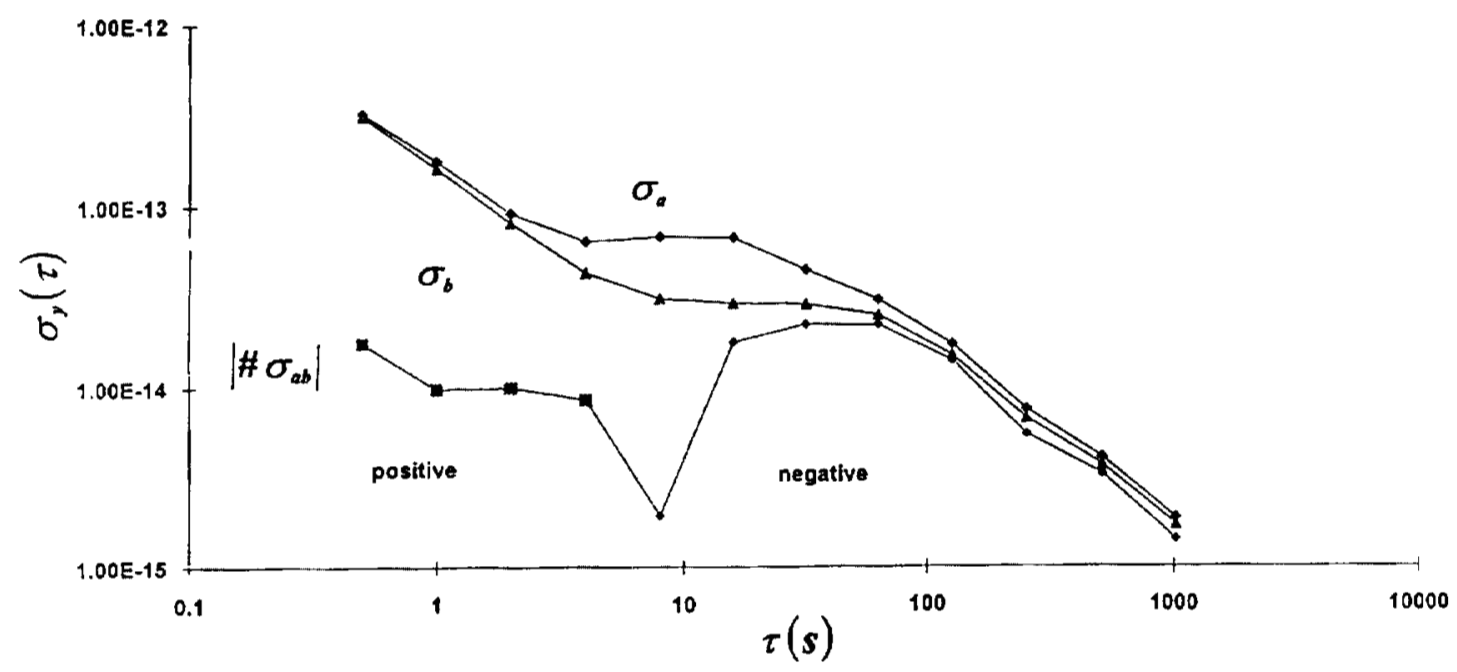

Figure 5. System noise floor for $\# \sigma_{y}(\tau)$ and $\sigma_{y}(\tau)$ after improving the measurement hardware. 


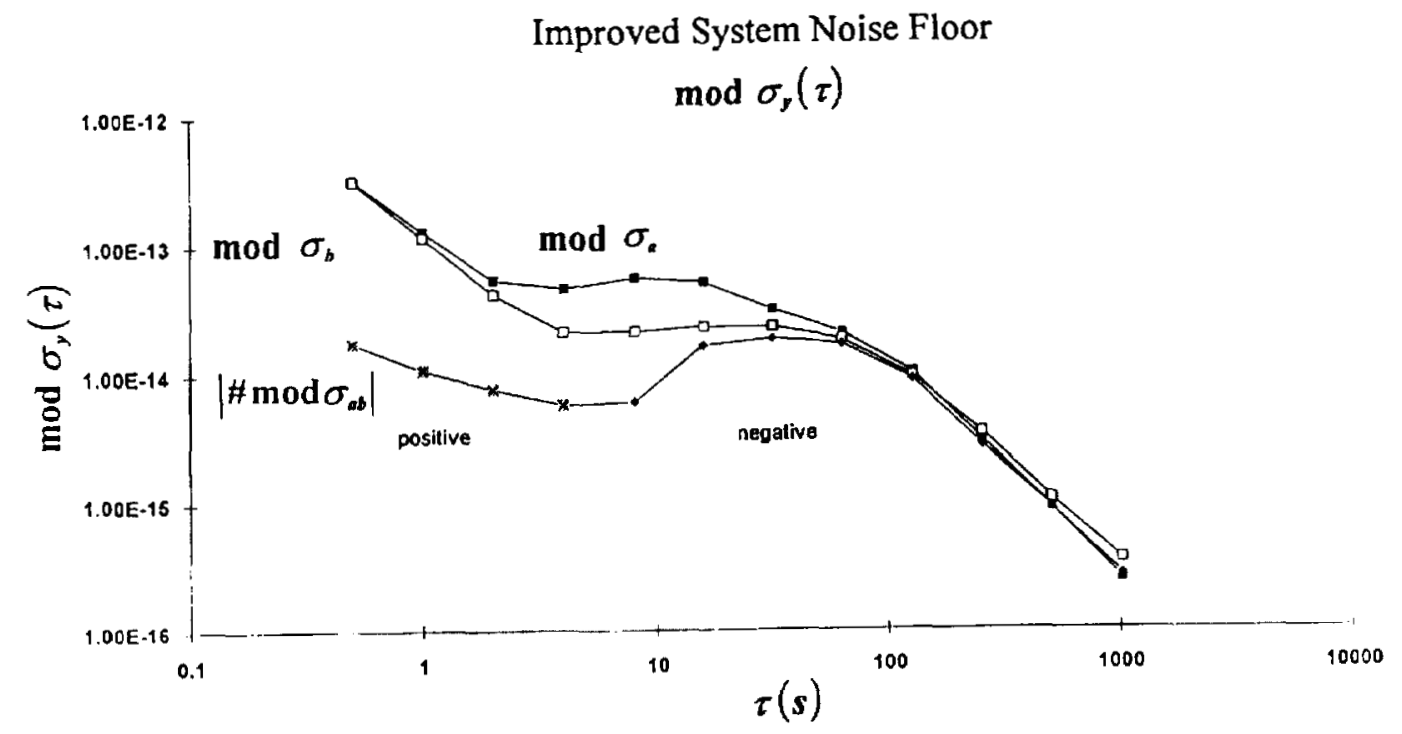

Figure 6. System noise floor for $\# \bmod \sigma_{\mathrm{y}}(\tau)$ and $\bmod \sigma_{\mathrm{y}}(\tau)$ after improving the measurement hardware.

\section{$\mathbf{R}^{2}$ Correlation for the \#mod $\sigma$ Noise Floor}

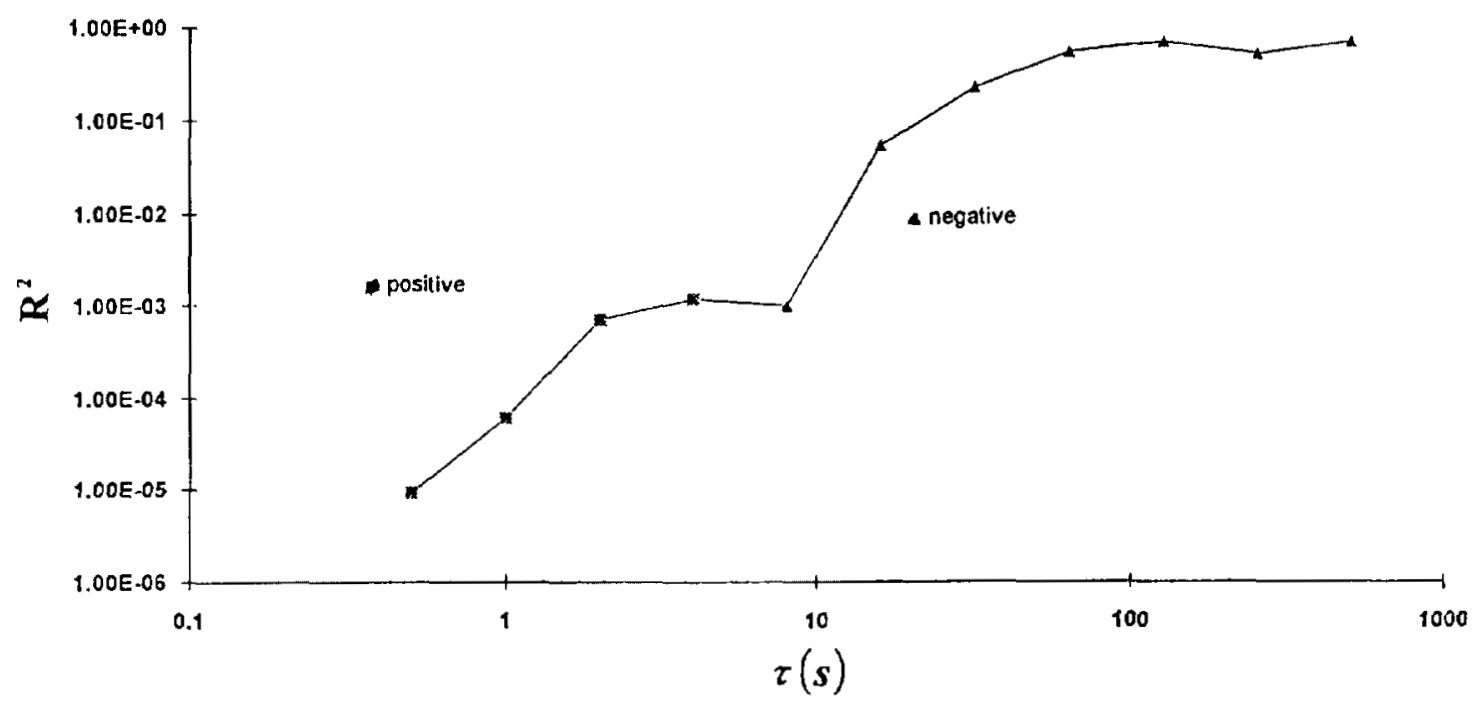

Figure 7. $\mathrm{R}^{2}$ for \#modo versus measurement time $\tau$. The increase in $\mathrm{R}^{2}$ at longer $\tau$ indicated increased correlated noise. The dips in $\mathrm{R}^{2}$ coincide with the change in the sign of \#mod $\sigma$ indicating a change in the correlation mechanism. 


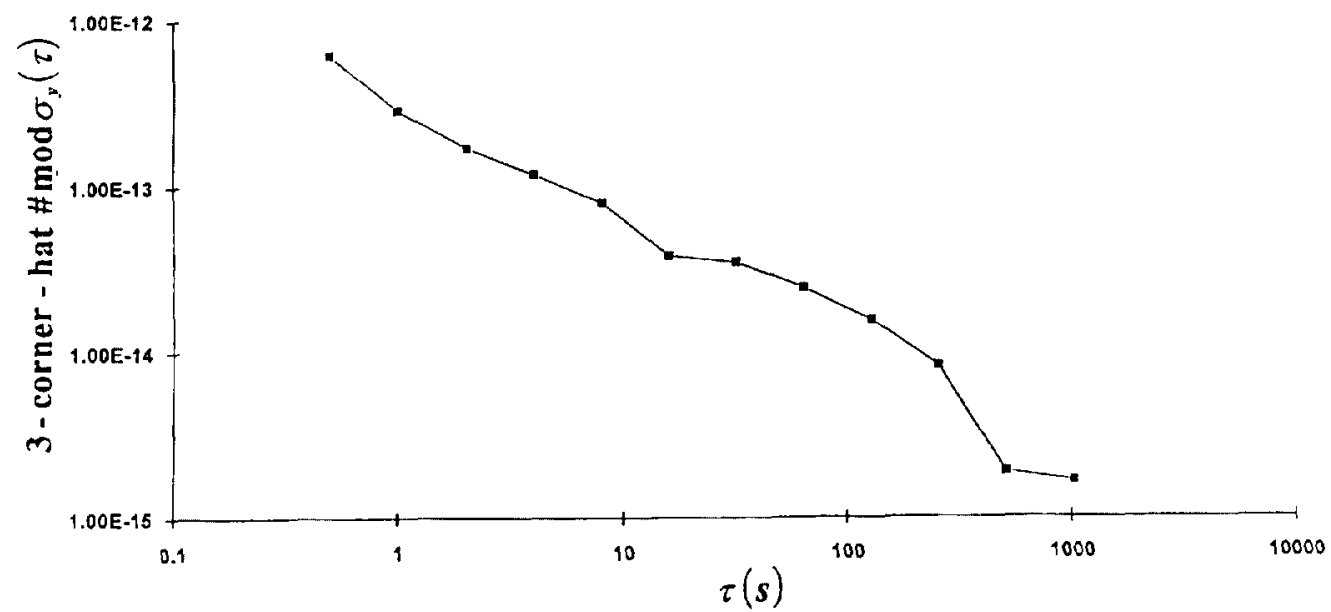

Figure 8. Estimates of \#mod $\sigma_{\mathrm{y}}(\tau)$ at $5 \mathrm{MHz}$ reference frequencies for an active hydrogen maser versus measurement time $\tau$.

\section{Conclusions}

We have shown the usefulness of using \# $\sigma$ and $R$ as tools to lower the noise floor resulting so far in an overall improvement of about 100 . These improvements enable low noise measurements that were not possible earlier at $5 \mathrm{MHz}$. We have shown as well that the $\# \sigma$ is a good estimate of the Allan and modified Allan variances and also some precise relationships concerning the 3-cornered-hat. Additional work should be done to further eliminate the common noise injected from the various sources to lower \#o. Once the tools are established they can be used to improve the present system to its limits and to design a new and better system. Other systems having separate measurement channels, such as the common view GPS, may benefit from these methods as well.

\section{Acknowledgments}

We gratefully acknowledge helpful discussion with David W. Allan, David A. Howe, and Marc A. Weiss.

\section{References}

[1] James A. Barnes, et al., "Characterization of Frequency Stability, IEEE Transactions on Instrumentation and Measurement, " IM$20,105-120,1971$.
[2] David W. Allan and James A. Barnes, "A Modified "Allan Variance" with Increased Oscillator Characterization Ability," Proc. 35th Annual Frequency Control Symposium, 470-475, 1981.

[3] D. W. Allan, M. A. Weiss and J. L. Jespersen, "A frequency-domain view of time domain characterization of clocks and time and frequency distribution systems," Proc. Annual Symposium on Frequency Control, 667-678, 1991.

[4] S. Stein, D. Glaze, J. Levine, J. Gray, D. Hilliard, D. Howe, "Performance of an Automated High Accuracy Phase Measurement System," Proc. 36th Annual Frequency Control Symposium, 314-320, 1982.

[5] D. J. Glaze and S. R. Stein, "Picosecond time difference measurements utilizing CAMAC based ANSI/IEEE -488 data acquisition hardware," NBS Tech Note 1056.

[6] J. Groslambert, D. Fest, M. Olivier, and J. J. Gagnepain, "Characterization of Frequency Fluctuations by Crosscorrelations and by Using Three or More Oscillators," Proc. 35th Annual Frequency Control Symposium, 458-463, 1981.

[7] J. F. Ratcliffe, Elements of Mathematical Statistics, Oxford University Press, 173-176, 1962. 